\title{
Belirli koşul altındaki asal gamma halkalarının tek yanlı idealleri ve türevleri üzerine
}

\author{
Ahmet GENÇ* \\ Adnan Menderes Üniversitesi Fen Edebiyat Fakültesi Matematik. Bölümü, Merkez Kampüsü, Aydın. \\ Geliş Tarihi (Recived Date): 11.09.2018 \\ Kabul Tarihi (Accepted Date): 16.01.2019
}

\section{Özet}

Bu çalışmada, belirli koşul altında $M$ asal gamma halkasının herhangi bir $(d, k)$ türevi için elde ettiğimiz bazı sonuçlar Brešar' in [3] “One-sided Ideals and Derivations of Prime Rings" adlı makalesine paralel olarak verilmiştir. Brešar makalesin de $R$ asal halka, $d, R$ halkasının bir türevi ve $U, R$ halkasının bir săg ideali olmak üzere, $d(U) U=0$ koşulu varken $q U=0$ olacak şekilde bazı $q \in Q$ için, $d(x)=[q, x]=q x-x q$ olduğunu ifade etmiş ve daha sonraki bazı teoremlerde bu özellikten faydalanmıştır. Bu makalede benzer karekterizasyonlar asal gamma halkalar için elde edilmeye çalışılmıştır.

Anahtar kelimeler: Asal gamma halka, türev, $(d, k)$ türev, să̆ ideal.

\section{On under the certain condition of one-sided ideals and derivations of prime gamma rings}

\begin{abstract}
In this study we obtained some results for under certain condition of prime gamma rings parallel to the study of Brešar [3] "One-sided Ideals and Derivations of Prime Rings". Brešar proved if $d(U) U=0$ then $d(x)=[q, x]=q x-x q$ for $q \in Q$ s.t. $q U=0$, for prime ring $R$ with Char $R \neq 2, d$, derivation on $R$ and $U$, right ideal of $R$. And use this for some next theorems. In this study we try to obtain similar characterization for prime gamma rings.
\end{abstract}

Keywords: Prime gamma ring, derivation, $(d, k)$ derivation, right ideal.

\footnotetext{
*Ahmet GENÇ, agenc@adu.edu.tr, http://orcid.org/0000-0002-2535-1689
} 


\section{Giriș}

Üçlü cebirsel yapı olarak gamma halkası kavramı ilk olarak 1964 yılında Nobusawa tarafından ortaya konmuştur. Bu kavram verilirken, $A$ ve $B$ toplamsal iki grup, $\operatorname{Hom}(A$, $B)$ ve $\operatorname{Hom}(B, A)$ sirasiyla $A$ dan $B$ ye ve $B$ den $A$ ya grup homomorfizmalarının kümesi olmak üzere, $\operatorname{Hom}(A, B) \times \operatorname{Hom}(B, A) \times \operatorname{Hom}(A, B)$ den $\operatorname{Hom}(A, B)$ toplamsal grubu içine $(f, \gamma, g) \rightarrow$ forog (fonksiyonların bileşke işlemi) ile tanımlanan üçlü çarpım model olarak alınmıştır. Nobusawa [13], gamma halkasının tanımını verdiği çalışmasında Wedderburn-Artin teoremini genelleştirmiştir.

1964 yılından günümüze kadar gamma halkalarının yapısı hakkında birçok çalışma yapılmıştır. Bu çalışmalar, gelecekte bu konunun çok önem kazanacağını ve üzerinde çok çalışılacağını ortaya koymaktadır. Gamma halkaları hakkında yapılan bazı çalışmalardan kısaca söz edilmesi, konunun boyutunu ortaya koyacaktır.

Barnes [1], $\Gamma$ ve $M$ toplamsal iki grup olmak üzere Nobusawa' nın verdiği gamma halka tanımında yer alan

$$
\begin{aligned}
& M \mathrm{x} \Gamma \mathrm{x} M \rightarrow M \quad \text { ve } \quad \Gamma \mathrm{x} M \mathrm{x} \Gamma \rightarrow \Gamma \\
& (m, \gamma, n) \rightarrow m \gamma n \quad \text { ve } \quad(\gamma, m, \beta) \rightarrow \gamma m \beta
\end{aligned}
$$

üçlü çarpımından ikincisini kaldırarak gamma halkası tanımını genelleştirmiştir. Aynı çalışmada, Barnes gamma halka homomorfizmasını tanımlamış ve gamma halkalar için homomorfizma teoremlerini vermiştir. Luh [11] asal gamma halka tanımını vermiştir. Kyuno [7, 9, 10] gamma halkalarının asal idealleri üzerine çalışmıştır. Ravinskar and Shukla [14] ve Kyuno [8] birbirinden bağımsız olarak gamma halkası üzerinde modül tanımını vermişlerdir.

Her Barnes anlamında $M, \Gamma$ - halkası Nobusawa anlamında $\Gamma^{\prime}$-halkası olacak şekilde bir toplamsal $\Gamma^{\prime}$ grubu bulunabileceğinden bu çalışmada Nobusawa anlamında $\Gamma$ halkaları incelenmiştir ve $(\Gamma, M)_{N}$ şeklinde gösterilmiştir.

Halka teoride yapılan çalışmalarda, Martindale Kesirler Halkası önemli bir rol oynamaktadır. Bu konu hakkındaki çalışmalar, Martindale [12] nin çalışması ile başlamıştır. Passman [15] ve Beidar, Martindale III and Mikhalev [2] bu teoriye önemli katkılarda bulunmuşlardır. Genç [5] yaptığı çalışmayla Nobusawa anlamında asal gamma halkalarında kesirler halkasını tanımlayıp, kesirler halkası için bazı karakterizasyonları vermiş ve gamma halkanın genişletilmiş merkezi tanımlanmıştır. Aynı çalışmada, Kandamar' in [6] tanımladığı $(d, k)$ türevi asal gamma halkalarının kesirler halkasına genişletilebileceği gösterilmiştir.

\section{2. Önbilgiler}

Bu çalışmada kullandığımız bazı kavramların tanımları aşağıda verilmiştir.

2.1 Tanım: $M$ elemanları $a, b, c, \ldots$ ile gösterilen toplamsal değişmeli grup ve $\Gamma$ elemanları $\alpha, \beta, \gamma, \ldots$. ile gösterilen toplamsal değişmeli grup olsun. Her $a, b \in M$ ve $\gamma$, 
$\beta \in \Gamma$ için, $a \gamma b \mathrm{M}$ toplamsal grubunun elemanı ve $\gamma a \beta$ elemanı da $\Gamma$ toplamsal grubunun elemanı olarak tanımlansın. Eğer bu çarpımlar aşağıdaki üç koşulu da sağlanıyor ise $\mathbf{M}$ toplamsal grubuna $\Gamma$-halkası denir.

(i) $\left(a_{1}+a_{2}\right) \gamma b=a_{1} \gamma b+a_{2} \gamma b$

$a\left(\gamma_{1}+\gamma_{2}\right) b=a \gamma_{1} b+a \gamma_{2} b$

$a \gamma\left(b_{1}+b_{2}\right)=a \gamma b_{1}+a \gamma b_{2}$

(ii) $(a \gamma b) \beta c=a \gamma(b \beta c)=a(\gamma b \beta) c$

(iii) Her $a, b \in M$ için $a \gamma b=0$ ise $\gamma=0$.

2.2 Tanım: $I \subseteq M$ olmak üzere, (Г,M) gamma halkasının, $I \Gamma M=\{a \alpha c \mid a \in I, \alpha \in \Gamma, c \in M\}$ $(M \Gamma I)$ kümesini kapsayan I toplamsal grubuna $(\Gamma, M)$ gamma halkasının sa $\breve{g}$ (sol) ideali denir. Eğer $I$ hem sağ hem de sol ideal ise $I$ toplamsal grubuna $(\Gamma, M)$ gamma halkasının iki yanlı ideali veya kısaca $(\Gamma, M)$ gamma halkasının ideali denir.

2.3 Tanım: $(\Gamma, \mathrm{M})_{\mathrm{N}}$ gamma halka olsun. Her $x \in M$ için, $e \delta x=x$ olacak şekilde $e \in M$ ve $\delta \in \Gamma$ var ise $(\delta, e)$ çiftine ( $\Gamma, \mathrm{M})$ gamma halkasının güçlü sol birimi denir. Her $x \in M$ için, $x \delta e=x$ olacak şekilde $e \in M$ ve $\delta \in \Gamma$ var ise $(\delta, e)$ çiftine $(\Gamma, M)$ gamma halkasının güçlï săg birimi denir. $(\delta, e)$ çifti, $(\Gamma, M)$ gamma halkasının hem güçlü sağ ve sol birim ise kısaca güçlü birim denir.

2.4 Tanım: $(\Gamma, M)$ gamma halka olsun. Eğer $(\Gamma, M)$ gamma halkasının herhangi iki $A$ ve $B$ ideali için, $A \Gamma B \subseteq P$ olması $A \subseteq P$ veya $B \subseteq P$ olmasını gerektiriyorsa, $(\Gamma, M)$ nın $P$ idealine asal ideal denir.

2.5 Tanım: $(\Gamma, M)$ gamma halkasının sıfır ideali asal ideal ise $(\Gamma, M)$ gamma halkasına asal gamma halka denir.

2.6 Tanım: Her $n, n_{1}, n_{2} \in N, m, m_{1}, m_{2} \in M$ ve $\alpha, \beta \in \Gamma$ için, aşağıdaki koşulları sağlayan $N$ toplamsal değişmeli grubuna săg $\Gamma M$ - modül denir.

(i) $n \alpha m \in N$

(ii) $\left(n_{1}+n_{2}\right) \alpha m=n_{1} \alpha m+n_{2} \alpha m$

(iii) $n(\alpha+\beta) m=n \alpha m+n \beta m$

(iv) $n \alpha\left(m_{1}+m_{2}\right)=n \alpha m_{1}+n \alpha m_{2}$

2.7 Tanım: $N_{1}$ ve $N_{2}$ sağ $\Gamma M-$ modül olsun. Aşağıdaki koşulları sağlayan

$\theta: N_{1} \rightarrow N_{2}$ dönüşümüne sağ $\Gamma \boldsymbol{M}$ - modül homorfizması denir.

(i) $\theta: N_{1} \rightarrow N_{2}$ grup homomorfizmasidir,

(ii) Her $x \in N_{1}$ ve $m \in M, \alpha \in \Gamma$ için, $\theta(x \alpha m)=\theta(x) \alpha m$.

2.8 Tanım: $M$, Barnes anlamında $\Gamma$-halka ve sirasiyla $d$ ve $k, M$ den $M$ e ve $\Gamma$ dan $\Gamma$ ya toplamsal dönüşümler olsun. Eğer her $a, b \in M$ ve $\beta \in \Gamma$ için, $d(a \beta b)=d(a) \beta b+a k(\beta) b+a \beta d(b)$ ise $d$ ye $(\Gamma, M)$ gamma halkasının $\boldsymbol{k}$-türevi denir.

$(\Gamma, M)_{\mathrm{N}}$ asal gamma halka ve $U$ sifırdan farklı $(\Gamma, M)_{\mathrm{N}}$ gamma halkasının bir ideali olsun. 
$\aleph=\{(U, f) \mid f: U \rightarrow M f$, sol $M \Gamma-$ modül homomorfizma $\}$

kümesi üzerinde, “ ” bağıntısı “( $U, f)\left.\sim(V, g) \Leftrightarrow f\right|_{W}=\left.g\right|_{W}$ olacak şekilde

$(\Gamma, M)_{\mathrm{N}}$ gamma halkasının $U \cap V$ tarafından kapsanan sıfırdan farklı en az bir $W$ ideali vardır" şeklinde tanımlansın. $\aleph$ kümesinin tüm elemanlarının denklik sınıflarının kümesi $Q$ olsun. $Q$ üzerinde "+" işlemi aşağıdaki gibi tanımlansın.

$\hat{f}+\hat{g}=\overline{(U, f)}+\overline{(V, g)}=\overline{(U \cap V, f+g)}$

Benzer şekilde, $(M, \Gamma)_{\mathrm{N}}$ asal gamma halka ve $0 \neq \Omega \quad(M, \Gamma)_{\mathrm{N}}$ gamma halkasının bir ideali olsun.

$\mathfrak{R}=\{(\Omega ; \tau) \mid \tau: \Omega \rightarrow \Gamma \tau$, sağ $M \Gamma-$ modül homomorfizması $\}$

$\Re$ kümesi üzerinde “ $\approx$ ” bağıntısı şu şekilde tanımlansın.

“( $\left.(\Omega, \tau) \approx(\Lambda, \sigma) \Leftrightarrow \tau\right|_{\Pi}=\left.\sigma\right|_{\Pi}$ olacak şekilde $(M, \Gamma)_{\mathrm{N}}$ gamma halkasının $\Omega \cap \Lambda$ tarafından kapsanan sıfırdan farklı en az bir $\Pi$ ideali vardır."

$\mathfrak{R}$ kümesinin bütün elemanlarının denklik sınıflarının kümesini $\Delta$ ile gösterelim. $\Delta$ üzerinde toplama işlemini aşağıdaki gibi tanımlayalım.

$\hat{\tau}+\hat{\sigma}=\overline{(\Omega, \tau)}+\overline{(\Lambda, \sigma)}=\overline{(\Omega \cap \Lambda, \tau+\sigma)}$

Q ve $\Delta$ toplamsal değişmeli grupları göz önüne alınarak aşağıdaki işlemini tanımlayalım;

. $Q \times \Delta \times Q \rightarrow Q$ işlemini,

$(\hat{f}, \hat{\tau}, \hat{g}) \rightarrow \hat{f} \cdot \hat{\tau} \cdot \hat{g}=\overline{(U, f)} \overline{(\Omega, \tau)} \overline{(V, g)}=\overline{(V \Omega U, f \stackrel{\Delta}{\tau} g)}$ ile tanımlayalım.

$(\Delta, Q)_{\mathrm{N}}$ gamma halkadır. Benzer şekilde aşağıdaki işlem altında $(Q, \Delta)_{\mathrm{N}}$ nın gamma halka olduğu görülebilir.

$(\hat{\tau}, \hat{f}, \hat{\sigma}) \rightarrow \hat{\tau} \cdot \hat{f} \cdot \hat{\sigma}=\overline{(\Omega, \tau)} \overline{(U, f)} \cdot \overline{(\Lambda, \sigma)}=\overline{(\Lambda U \Omega, \tau \hat{f} \sigma)}$ olacak şekilde,

$. \Delta \times Q \times \Delta \rightarrow \Delta$ “.” işlemi tanımlanır ve burada $\stackrel{\Delta}{f}: \Gamma U \Gamma \rightarrow \Gamma$

$(\gamma m \beta) \stackrel{\Delta}{f}=\gamma f(m) \beta$ olarak tanımlanır.

2.9 Tanım: $(\Delta, Q)_{\mathrm{N}}$ gamma halkasına $(\Gamma, M)_{\mathrm{N}}$ gamma halkasının kesirler halkası denir.

\section{Belirli koşul altında türev için bazı sonuçlar}

$(\Gamma, M)_{\mathrm{N}}$ asal gamma halka olmak üzere, $(\Gamma, M)_{\mathrm{N}}$ gamma halkası $M \gamma a=0$ ise $a=0$ koşulunu sağlasın. Özel olarak, $(\varepsilon, e)(\Gamma, M)_{\mathrm{N}}$ gamma halkasının sol birimi ise $\gamma$ yerine $\varepsilon$ alınarak $M \gamma a=0$ ise $a=0$ koşulunun sağlandığı görülür. $U,(\Gamma, M)_{\mathrm{N}}$ gamma 
halkasının sıfırdan farklı ideali ve $L \gamma(U)=\{x \in M \mid x \gamma U=0, \gamma \in \Gamma\}$ olsun. Çalışmanın bundan sonraki kısımlarında $L \gamma(U)$ gösterimi yerine kısaca $L \gamma$ kullanılacaktır. Aşağıdaki örnekte sıfırdan farklı asal gamma halkada, $k(\gamma)=0$ olacak şekilde, sıfırdan farklı bir $\gamma$ elemanı bulunabileceği gösterilmiştir.

3.1. Örnek $M=\left\{\left[\begin{array}{ll}a & b \\ c & d\end{array}\right]: a, b, c, d \in \mathbb{Z}\right\}=M_{2}(\mathbb{Z})$ ve $\Gamma=\left\{\left[\begin{array}{ll}e & f \\ g & h\end{array}\right]: e, f, g, h \in \mathbb{Z}\right\}$ olmak üzere, $(\Gamma, M)_{N}$ asal gamma halkadır. $k: \Gamma \rightarrow \Gamma k(\gamma)=[\beta, \gamma]_{a}=\beta a \gamma-\gamma a \beta$ iç türevi için, $\gamma=\left[\begin{array}{ll}0 & 1 \\ 0 & 0\end{array}\right], \beta=\left[\begin{array}{ll}0 & 0 \\ 0 & 1\end{array}\right]$ ve $a=\left[\begin{array}{ll}1 & 0 \\ 0 & 0\end{array}\right]$ alırsak, $k(\gamma)=0$ olur.

3.2. Özellik $U,(\Gamma, M)_{\mathrm{N}}$ gamma halkasının sıfırdan farklı bir ideali ve $(d, k),(\Gamma, M)_{\mathrm{N}}$ gamma halkası üzerinde bir türev olsun. Eğer $d(U) \subseteq L \gamma$ (yani $d(U) \gamma U=0$ ) ise $k(\gamma)=0$ dir.

İspat: Her $u, w \in U$ için, $d(u) \gamma w=0$ olsun. $d(u) \gamma w=0$ eşitliğinde $u$ yerine $u \gamma v$ koyarsak, $0=d(u \gamma v) \gamma w=d(u) \gamma v \gamma w+u k(\gamma) v \gamma w+u \gamma d(v) \gamma w, \forall u, v, w \in U$ elde edilir. $d(U) \gamma U=0$ olduğundan, $0=u k(\gamma) v \gamma w, \forall u, v, w \in U$ olur. $(\Gamma, M)_{\mathrm{N}}$ asal gamma halka olduğundan sabit bir $\gamma \in \Gamma$ için, $k(\gamma)=0$ bulunur.

3.3. Özellik Kabul edelim ki $0 \neq U$ ve $k(\gamma)=0$ olsun. Aşağıdaki koşullar denktir;

(i) $d(U) \subseteq L \gamma$

(ii) $d(U) \gamma d(U)=0$

(iii) $d(U) \gamma a=0$ olacak şekilde en az bir sıfırdan farklı $a \in M$ vardır.

(iv) $q \gamma U=0$ olacak şekildeki bazı $q \in Q$ için, $d(x)=[q, x]=q \gamma x-x \gamma q$ dir.

İspat (i $\Rightarrow$ ii) $: d(U) \gamma U=0$ olsun. Her $\beta \in \Gamma$, her $u, v, w \in U$ için, $0=d(u \beta d(v)) \gamma w=(d(u) \beta d(v)) \gamma w+(u k(\beta) d(v)) \gamma w+\left(u \beta d^{2}(v)\right) \gamma w$

Hipotezden, $u \beta d^{2}(v) \gamma w=0 \forall \beta \in \Gamma, \forall u, v, w \in U$.

$(\Gamma, M)_{\mathrm{N}}$ asal gamma halka olduğundan,

$d^{2}(v) \gamma w=0, \forall v, w \in U$

Hipotezden ve Özellik 3.2 ve (1) eşitliği kullanılarak,

$0=d(d(u) \gamma w)=d^{2}(u) \gamma w+d(u) k(\gamma) w+d(u) \gamma d(w)$

$$
=d(u) \gamma d(w) \forall u, w \in U
$$

elde edilir. Böylece $d(U) \gamma d(U)=0$ olur.

(ii $\Rightarrow$ iii) : Asal gamma halkanın sıfırdan farklı bir ideali üzerinde sıfırdan farklı bir türev yok olmaz. Eğer $d \neq 0,0 \neq U \triangleleft M$ ise $d(U) \neq 0 . d(U)=0$ olsun.

$0=d(u \beta v)=d(u) \beta v+u k(\beta) v+u \beta d(v)=u k(\beta) v \forall u, v \in U, \forall \beta \in \Gamma$.

$U \triangleleft M$ ve $(\Gamma, M)_{\mathrm{N}}$ asal gamma halka olduğundan, $0=m k(\beta) n \forall m, n \in M, \forall \beta \in \Gamma$ elde edilir. $(\Gamma, M)_{\mathrm{N}}$ gamma halka olması kullanılarak, her $\beta \in \Gamma$ için, $k(\beta)=0 \forall \beta \in \Gamma$. Yani $k=0$.

Bu yüzden, $0=d(u \beta m)=d(u) \beta m+u k(\beta) m+u \beta d(m) \forall m \in M, \forall \beta \in \Gamma, \forall u \in U$.

Hipotezden ve $k=0$ eşitliğinden $u \beta d(m)=0$ olur. $(\Gamma, M)_{\mathrm{N}}$ gamma halka asallığından 
$d(M)=0$ elde edilir. Bu da çelişkidir. Öyleyse, eğer $d(U) \gamma d(U)=0$ ise bazı sıfırdan farklı $a \in M$ için, $d(U) \gamma a=0$ dır.

(iii $\Rightarrow$ i) : Bazı sıfırdan farklı $a \in M$ için $d(U) \gamma a=0$ olsun.

$0=d(u \gamma v) \gamma a=d(u) \gamma v \gamma a+u k(\gamma) v \gamma a+u \gamma d(v) \gamma a \forall u, v \in U . k(\gamma)=0$ olmasi kullanilarak $d(u) \gamma v \gamma a=0, \forall u, v \in U$ elde edilir. ( $\Gamma, M)_{\mathrm{N}}$ gamma halkasının asallığından, $d(u) \gamma v=0$ veya $m \gamma a=0 \forall u, v \in U, \forall m \in M$ olur. Eğer her $m \in M$ için, $m \gamma a=0$ ise $M$ üzerindeki hipotezden, $a=0$. Bu da $a$ nın sıfırdan farklı olması ile çelişir. O zaman, $d(u) \gamma v=0$, $\forall u, v \in U$.

(iii $\Leftrightarrow$ iv) : İspat Herstein' nın [4]çalışmasına paralel olarak verilmiştir. $u \in U, x \in M$ ve $\beta \in \Gamma$ olsun. $U,(\Gamma, M)_{\mathrm{N}}$ gamma halkasının ideali olduğundan, $u \beta x \in U$ elde edilir. $d(u) \gamma a=0$ eşitliğinde $u$ yerine $u \beta x$ koyarsak

$0=d(u \beta x) \gamma a=d(u) \beta x \gamma a+u k(\beta) x \gamma a+u \beta d(x) \gamma a, \forall u \in U, \forall x \in M, \forall \beta \in \Gamma$ elde ederiz.

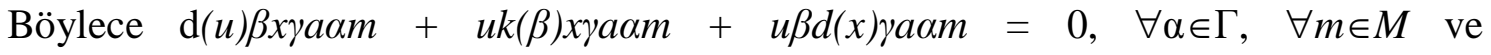
$d(u) \beta\left(\sum_{\text {sonlu }} x \gamma a \alpha m\right)=-u \beta\left(\sum_{\text {sonlu }} d(x) \gamma a \alpha m\right)-u k(\beta) x \gamma a \alpha m$ olur. $\mathrm{Bu}$ yüzden, herhangi $v \in V=M \gamma a \Gamma M$ için, burada $V,(\Gamma, M)_{\mathrm{N}}$ gamma halkasının sıfırdan farklı idealidir. (Ĕger $V=0$ ise $M=0$ veya $M \gamma a=0$. Bu da çelişkidir.)

$d(u) \beta v=u \beta f(v)-u k(\beta) v, \forall \beta \in \Gamma, \forall u, v \in U$

$f(v) u$ dan bağımsız fakat $v$ elemanına bağımlıdır. $M$ asal gamma halka olduğundan, $f(v)$ iyi tanımlı ve her $v \in V$ için teklikle belirlidir. Her $y \in M, v \in V$ ve $\alpha \in \Gamma$ için, $v \alpha y \in V$ dır. (2) eşitliğinde $v$ yerine $v \alpha y$ koyarsak,

$d(u) \beta(v \alpha y)=u \beta f(v \alpha y)-u k(\beta) v \alpha y$

elde edilir. (2) ve (3) eşitliklerini kullanarak,

$(d(u) \beta v) \alpha y)=u \beta f(v) \alpha y-u k(\beta) v \alpha y=u \beta f(v \alpha y)-u k(\beta) v \alpha y$

$\Rightarrow u \beta f(v) \alpha y=u \beta f(v \alpha y)$

$\Rightarrow u \beta[f(v) \alpha y-f(v \alpha y)]=0, \forall u \in U, \forall \beta \in \Gamma$ elde edilir. O zaman, $M$ nin asallığından,

$f(v) \alpha y=f(v \alpha y), \forall v \in U, \forall \alpha \in \Gamma, \forall y \in M$

olur. Bu da $f: V \rightarrow M$ dönüşümünün sağ $\Gamma M$ - modül homomorfizması olduğunu gösterir. Bu durumda, $q=\overline{(V, f)} \in Q$ olur. Buna ilaveten, Özellik 3.5 .1 (i) [7] şıkkından her $v \in V$ için, $f(v)=q \gamma v$ elde edilir. $x \in M, v \in V$ ve sabit $\gamma \in \Gamma$ için, (2) eşitliğinde $v$ yerine $x \gamma v$ koyarak,

$d(u) \beta x \gamma v=u \beta f(x \gamma v)-u k(\beta) x \gamma v=u \beta q \gamma x \gamma v-u k(\beta) x \gamma v$

(2) eşitliğinde $u$ yerine $u \gamma x$ koyarak,

$d(u \gamma x) \beta v=u \gamma x \beta f(v)-u \gamma x k(\beta) v=u \gamma x \beta q \gamma v-u \gamma x k(\beta) v$

ve $d(u \gamma x) \beta v=d(u) \gamma x \beta v+u k(\gamma) x \beta v+u \gamma d(x) \beta v$ elde edilir. (5) eşitliği ve $k(\gamma)=0$ kullanılarak, 
$d(u \gamma x) \beta v=u \gamma q \beta x \beta v+u \gamma d(x) \beta v$

bulunur. (6) ve (7) eşitliklerini kullanarak da

$u \gamma x \beta q \gamma v-u \gamma x k(\beta) v=u \gamma q \beta x \beta v+u \gamma d(x) \beta v \quad \forall \beta \in \Gamma$

elde edilir. (8) eşitliğinde $\beta$ yerine $\gamma$ koyarak $u \gamma x \gamma q \gamma v-u \gamma x k(\gamma) v=u \gamma q \gamma x \gamma v+u \gamma d(x) \gamma v$ ve $\mathrm{k}(\gamma)=0$ kullanilarak, $u \gamma[x \gamma q-q \gamma x-d(x)] \gamma v=0 \quad \forall u, v \in U$ bulunur. $(\Gamma, M)_{\mathrm{N}}$ gamma halkasının asallığı ve $M$ üzerindeki hipotezden, $d(x)=q \gamma x-x \gamma q, \forall x \in M$ elde edilir.

Tersine, $q \gamma U=0$ olacak şekildeki bazı $q \in Q$ için, $d(x)=[q, x]=q \gamma x-x \gamma q$ olsun. $x \in U$ alınırsa $[q, x] \gamma v=(q \gamma x-x \gamma q) \gamma v=q \gamma x \gamma v-x \gamma q \gamma v=0$ bulunur. O halde, (iii) şıkkındaki $a$ elemanını $U$ idealinden sıfırdan farklı bir elaman alarak sağlandığı görülür.

3.4. Teorem $(\Gamma, M)_{N}$ gamma halkasının karakteristiği ikiden farklı ve $k(\gamma)=0$ olsun. O zaman $d(U)$ ile üretilen alt halkanın $(\Gamma, M)_{\mathrm{N}}$ gamma halkasının sifirdan farklı ideallerini içermemesi için gerek ve yeter bir koşul $d(U) \subseteq L \gamma$ olmasıdır.

İspat: $A, d(U)$ ile üretilen alt halka ve $S=A \cap U$ olsun. $u \in U, s \in S$ ve sabit bir $\gamma \in \Gamma$ için, $d(s \gamma u)=d(s) \gamma u+s k(\gamma) u+s \gamma d(u)$ elde edilir. $k(\gamma)=0$ olduğundan ve $d(s \gamma u) \in A$, $s \gamma d(u) \in A$ olmasindan, $d(s) \gamma u \in A, \forall s \in S, \forall u \in U$ elde edilir. $d(S) \gamma U,(\Gamma, M)_{\mathrm{N}}$ gamma halkasının sağ ideali olur. $d(U)$ ile üretilen alt halka $(\Gamma, M)_{\mathrm{N}}$ gamma halkasının sıfırdan farklı idealini içermediğinden $d(S) \gamma U=(0)$ olmalıdır. $d(u \gamma a)=d(u) \gamma a+u k(\gamma) a+u \gamma d(a), k(\gamma)=0$ ve $d(u \gamma a) \in A, d(u) \gamma a \in A$ ve $U \triangleleft M$ olmasi kullanilarak $u \gamma d(a) \in S, \forall u \in U, \forall a \in A$ bulunur.

Bu yüzden, $0=d(u \gamma d(a)) \gamma v=d(u) \gamma d(a) \gamma v+u k(\gamma) d(a) \gamma v+u \gamma d^{2}(a) \gamma v, \forall u \in U, \forall a \in A$ elde edilir. $k(\gamma)=0$ olması kullanılarak,

$0=d(u) \gamma d(a) \gamma v+u \gamma d^{2}(a) \gamma v, \quad \forall u, v \in U, \forall a \in A$

bulunur. (9) eşitliğinde $u$ yerine $u \gamma w$ koyarsak,

$0=d(u \gamma w) \gamma d(a) \gamma v+u \gamma w \gamma d^{2}(a) \gamma v$

$=d(u) \gamma w \gamma d(a) \gamma v+u k(\gamma) w \gamma d(a) \gamma v+u \gamma d(w) \gamma d(a) \gamma v+u \gamma w \gamma d^{2}(a) \gamma v$

$k(\gamma)=0$ ve (9) eşitliğinden, $d(u) \gamma w \gamma d(a) \gamma v=0, \forall u, v, w \in U, \forall a \in A$

$(\Gamma, M)_{\mathrm{N}}$ gamma halkasının asallığı ve $U \triangleleft M$ olmasından $d(U) \gamma U=0$ veya $M \gamma d(A) \gamma U=0$ elde edilir. $M \gamma d(A) \gamma U=0$ olsun. $(\Gamma, M)_{\mathrm{N}}$ gamma halkasının üzerindeki hipotezden,

$d(A) \gamma U=0 \Rightarrow d^{2}(u) \gamma w=0, \forall u, w \in U$

bulunur. (10) eşitliğinde $u$ yerine $u \gamma v$ yazarsak

$0=d^{2}(u \gamma v) \gamma w=d(d(u) \gamma v) \gamma w+d(u k(\gamma) v) \gamma w+d(u \gamma d(v)) \gamma w$ elde edilir. $k(\gamma)=0$ den,

$0=d(d(u) \gamma v) \gamma w+d(u \gamma d(v)) \gamma w$

$\left.=d^{2}(u) \gamma v \gamma w+d(u) k(\gamma) v \gamma w+d(u) \gamma d(v) \gamma w+d(u) \gamma d(v) \gamma w+u k(\gamma) d(v)\right) \gamma w+u \gamma d^{2}(v) \gamma w$

$k(\gamma)=0$ ve (10) eşitliği kullanılarak, $0=2(d(u) \gamma d(v)) \gamma w \quad \forall u, v, w \in U$ bulunur. $(\Gamma, M)_{\mathrm{N}}$ gamma halkasının karakteristiği ikiden farklı olduğundan, 
elde edilir. (11) eşitliğinde $v \quad$ yerine $v \gamma t$ yazılarak, $0=d(u) \gamma d(v \gamma t) \gamma w=d(u) \gamma d(v) \gamma t \gamma w+d(u) \gamma v k(\gamma) t \gamma w+d(u) \gamma v \gamma d(t) \gamma w$ olur. $k(\gamma)=0$ ve (11) eşitliği kullanılarak, $0=d(u) \gamma v \gamma d(t) \gamma w, \forall u, v, w, t \in U$ bulunur. $(\Gamma, M)_{\mathrm{N}}$ gamma halkasının asallığı ve $(\Gamma, M)_{\mathrm{N}}$ gamma halkasının üzerindeki hipotezden, $d(U) \gamma U=0$ elde edilir.

Tersine, $d(U) \gamma U=0$ olsun. Öyleyse $A \gamma U=0$ olur. $(\Gamma, M)_{\mathrm{N}}$ gamma halkasının asallığ ve $(\Gamma, M)_{\mathrm{N}}$ gamma halkasının üzerindeki hipotezden, $A=0$ elde edilir yani $d(U)$ ile üretilen alt halka $(\Gamma, M)_{\mathrm{N}}$ gamma halkasının sıfırdan farklı idealini içermez.

3.5. Teorem $(\Gamma, M)_{\mathrm{N}}$ gamma halkası karakteristiği ikiden farklı ve yukarıdaki koşulu sağlayan asal gamma halka olsun. $U$, sıfırdan farklı $(\Gamma, M)_{\mathrm{N}}$ gamma halkasının sıfırdan farklı bir ideali, $\left(d_{1}, k_{1}\right)$ ve $\left(d_{2}, k_{2}\right), M$ nin sifırdan farklı iki türevi olsun. Eğer $d_{1} d_{2}(U)=(0)$ ise $q \Gamma U=(0)$ ve $p \Gamma U=(0)$ olacak şekilde $Q$ nun $p$ ve $q$ elemanları vardır.

İspat: Eğer $d_{1} d_{2}(U)=(0)$ ise $A, d_{2}(U)$ ile üretilen alt halka olmak üzere $d_{1}(A)=(0)$ dır. $d \neq 0$ ve $A$ sıfırdan farklı $(\Gamma, M)_{\mathrm{N}}$ gamma halkasının idealini içermediğinden, Teorem 3.4 gereğince her $u, v \in U$ ve sabit $\gamma \in \Gamma$ için, $d_{2}(u) \gamma v=0$ elde edilir. Özellik 3.3 den de $q \gamma U=(0) \quad$ olacak şekilde $q \in Q \quad$ vardır. $\mathrm{Bu}$ yüzden, $d_{2}(u \gamma v)=d_{2}(u) \gamma v+u k_{2}(\gamma) v+u \gamma d_{2}(v) . k(\gamma)=0$ ve $d_{2}(u) \gamma v=0$ olmasi kullanilarak, her $u, v \in U$ için, $d_{2}(u \gamma v)=u \gamma d_{2}(v)$ elde edilir. Bu durumda, $d_{2}(U) \neq 0$ olduğundan, $0=d_{1} d_{2}(u \gamma v)=d_{1}\left(u \gamma d_{2}(v)\right)=d_{1}(u) \gamma d_{2}(v) \forall u, v \in U$ bulunur. Böylece Özellik 3.3 kullanılarak $d_{1}(U) \gamma U=0$ ve $p \gamma U=(0)$ olacak şekilde $p \in Q$ var olduğu elde edilir.

\section{Sonuç}

Bu çalışmada, birimli veya $M \gamma a=0$ ise $a=0$ koşulunu sağlayan ve karakteristiği 2 den farklı, $(\Gamma, M)_{\mathrm{N}}$ asal gamma halkaları için, Brešar' in [3] çalışmasında verdiği karekterisazyonlara benzer şekilde, 3.3 Özellik, 3.4 ve 3.5 Teoremlerde verilen karekterizasyonlar elde edilmiştir.

\section{Kaynaklar}

[1] Barnes, W. E., On the $\Gamma$-ring of Nobusawa, Pacific Journal of Mathematics, 18, 411-422, (1966).

[2] Beidar K. I., Martindale III W. S. and Mikhalev, A. V., Rings with Generalized Identities, Marcel Dekker Incoporated, (1996).

[3] Brešar, M., One-Sided Ideals and Derivations of Prime Rings, Proceedings of the American Mathematical Sociaty, 122(4), 979-983 (1994).

[4] Herstein I.N., A Condition that a Derivation be Inner, Rendiconti Del Circolo Matematico Di Palermo, Serie II, 5-7 (1988).

[5] Genç A., Asal Gamma halkalarının Kesirler Halkası, Doktora Tezi, Ege Üniversitesi, Fen Bilimleri Enstitüsü, İzmir, (2008).

[6] Kandamar, H., The $k$-Derivation of a Gamma-Ring, Turkish Journal of Mathemtics, 23 (3), 221-229, (2000). 
[7] Kyuno, S., Gamma Rings, Hadronic Press Incorporated, (1991).

[8] Kyuno, S., On the Semi-simple Gamma Rings, Tohoku Mathematical Journal, 29, 217-225, (1977).

[9] Kyuno, S., On Prime Gamma Ring, Pacific Journal of Mathematics, 75, 185190, (1978).

[10] Kyuno, S., Prime Ideals in Gamma Rings, Pacific Journal of Mathematics, 98(2), 375-379, (1982).

[11] Luh, L., The Structure of Primitive Gamma Rings, Osaka Journal of Mathematics, 7 , 267-274, (1970).

[12] Martindale, W., Prime Rings Satisfying a Generalized Polynomial Identity, Journal of Algebra, 12, 576-584, (1969).

[13] Nobusawa, N., On a Generalization of the Theory, Osaka Journal of Mathematics, 1, 81-89, (1964).

[14] Ravinskar T. S. and Shukla U. S., Structure of $\Gamma$-rings. Pacific Journal of Mathematics, 80, 537-559, (1979).

[15] Passman, D., Infinite Crossed Products, Academic Press, San Diego, (1989). 